
3
Research Square
Preprints are preliminary reports that have not undergone peer review.
They should not be considered conclusive, used to inform clinical practice,
or referenced by the media as validated information.

\title{
How Different Health Literacy Dimensions Influences Health Status and Well- being among Men and Women: The Mediating Role of Health Behaviors
}

Fan Zhang

Education University of Hong Kong https://orcid.org/0000-0002-7580-5781

Peggy Pui-Lai Or ( $\sim$ peggyor@yahoo.com )

The Education University of Hong Kong https://orcid.org/0000-0001-6611-2724

Joanne Wai-Yee Chung

Education University of Hong Kong

\section{Research article}

Keywords: sex, health literacy dimensions, health behavior, physical condition, well-being

Posted Date: August 28th, 2020

DOI: https://doi.org/10.21203/rs.3.rs-23168/v2

License: (c) (i) This work is licensed under a Creative Commons Attribution 4.0 International License. Read Full License 


\section{Abstract}

Background. Health literacy, the ability to access, understand, evaluate, and apply health information, was found to contribute to positive health outcomes, possibly via promoting healthy behaviors. However, the specific pathways linking different health literacy skills to health and well-being has remained unclear.

Methods. A cross-sectional survey with structural questionnaires was administered among 2236 community-based adults in Hong Kong (mean age $=46.10$ \pm 19.05). Health literacy was measured by HLS-Asian-47. Participants' health behavior, physical conditions, and subjective well-being were reported.

Results. With structural modeling path analysis, health literacy in finding and understanding information showed a direct effect on enhancing physical health. While applying information capacity had an indirect positive effect via promoting health behaviors, which was moderated by sex. Only among women, this indirect effect predicting fewer physical symptoms and better well-being was significant.

Conclusions. Although similar patterns were found in physical condition and well-being, distinct direct and indirect pathways were found of different health literacy dimensions for men and women. Based on the findings, by targeting specific health literacy skills, education programs should be developed to enhance women's health knowledge, and men's application of the knowledge in healthy lifestyle.

\section{Background}

Health literacy, a concept referring to a set of abilities to access, comprehend, appraise, and apply information to function effectively to promote and maintain health in different contexts (Sørensen et al. 2012), was found to play a key role in individual's health behaviors and health status (Baker et al. 2007; Von Wagner et al. 2009). However, across different countries, the prevalence of inadequate health literacy was considerably high. For example, the Agency for Healthcare Research and Quality (AHRQ) has found that approximately one third of Americans only have limited health literacy, and this rate went up to 70\% in the age group of 75 and above (Koenig 2019). A recent systematic review on the prevalence of limited health literacy in Southeast Asia has reported that with a large variation across five countries (i.e., Laos, Malaysia, Myanmar, Singapore, and Thailand), on average, over 50\% of the population showed limited health literacy (Rajah et al. 2019), and the rate was even higher in healthcare settings (67.5\%). Similar results was reported in Hong Kong population by a recent study (Zhang et al. 2020), and the prevalence of limited health literacy was even higher in mainland China. In a sample of 1360 participants (aged 1569 ) in Shanghai, the prevalence of limited health literacy was approximately $85 \%$. In consistent, when looking at the health literacy on certain types of disease, over $70 \%$ of people showed limited health literacy about chronic disease (Qian et al. 2019); while about $80 \%$ do not have adequate health literacy for infectious disease in mainland China (Gao et al. 2018).

Why the prevalence of limited health literacy could be so high in Chinese society? It is possible that the general public has remained unaware about the significance and effects of health literacy. In fact, previous literature showed that greater health literacy was consistently associated with various benefits for individual's health, including more healthcare actions (Von Wagner et al. 2009), better health status (Baker et al. 2007), and greater subjective well-being (Angner et al. 2010). A systematic review reported that limited health literacy was associated with poorer physical health and higher all-cause mortality rate even after controlling for cognitive functioning (Berkman et al. 2011). Inadequate health literacy could lead to lower medical adherence among patients with cardiovascular disease (Murray et al. 2004), poorer glycemic control in type-2 diabetes (Schillinger et al. 2002), and higher hospital admission (Baker et al. 2002).

In addition to the effect on physical health, health literacy was also found to affect individual's mental health (Coffman and Norton 2010; Lincoln et al. 2006), although the existing evidence has been relatively thin. In the review by Berkman et al., (2011) only one study showed low health literacy was related to more depressive symptoms after controlling for the possible confounders (Lincoln et al. 2006). By investigating the relationship between health literacy and happiness, Angner et al., (2010) found inadequate health literacy, in addition to poverty and poor health, was associated with lower level of happiness. However, despite this finding being widely cited, the single-item measurement for health literacy (i.e., "how confident are you in filling out medical forms by yourself") may not accurately capture individual's ability to process health-related information, and happiness doesn't equal to well-being. Therefore, the current study would address the effects of health literacy on both subjective well-being and physical health.

Another research objective of the current study is to test the underlying mechanism of health literacy. It was proposed that health literacy may benefit health via promoting people's healthy lifestyle and behaviors. Unhealthy lifestyle behaviors, such as smoking, alcohol consumption, or being physically inactive, could lead to poorer health and higher mortality rate, and are directly linked to the top five causes of death (i.e., heart disease, cancer, cerebrovascular disease, respiratory disease, and diabetes, Mokdad et al. 2004). Fortunately, these risk behaviors were modifiable by certain psychosocial factors such as health literacy. With higher level of health literacy, people are more likely to engage in healthy lifestyles, including having frequent physical exercise (Suka et al. 2015), reducing the usage of alcohol, as well as getting regular physical examination, etc (Blow et al. 2000; Södergren et al. 2012). However, regarding the relationship between health literacy and dietary habits (Geboers et al. 2014) or smoking behavior (Geboers et al. 2016; Reisi et al. 2014), the finding was rather inconclusive. For example, Geboers et al., (2016) has analyzed the data of 3,241 older adults from the LifeLines Cohort Study, and found inadequate health literacy was associated with poorer health habits such as limited physical activities, insufficient intake of fruit and vegetables, and low alcohol use, but not smoking. The mixed findings could be a result of methodological inconsistency of health literacy research; also, given the multifaceted nature of health literacy, different dimensions may affect health behaviors differently. For example, it was found that the capacities of accessing to, comprehending, and applying health-related information were more important for smoking cessation among college students (Panahi et al. 2017), Therefore, instead of general health literacy, the current study examines the relationships between different dimensions of health literacy and health outcomes.

In addition, we also aim to address the potential gender difference in the effects of health literacy. Past research has found women's life expectancy is usually 4-5 years longer than men, although they are less healthy than men at any age (Bird and Rieker 1999). This could be an outcome of complex biological and social processes. When including gender difference in health behaviors (e.g., consumptions of tobacco, alcohol, or drug) into the economic model of health 
deficit accumulation, Schünemann et al., (2017) has found $89 \%$ of the gender gap in life expectancy was explained additionally. The behavioral difference could be mainly driven by the gender difference in health literacy (Sudore et al. 2006; Von Wagner et al. 2009). In particular, the level of health literacy was usually lower among men than women (for a review, see Davey et al. 2015), despite their tendency to over-report the abilities when answering health literacy questions (Lee et al. 2013a). Older women were more likely to watch health-related television programs and have social engagement, which may result in higher health literacy and better health (Duong et al. 2018). However, how one's sex would moderate the effects of health literacy on health behavior and health status has been relatively unstudied.

With a sample of 2236 community-dwelling individuals, the current study has a three-fold research purpose: 1) to explore how the effects of different health literacy skills would be mediated by health behaviors; 2) to test whether the mediation pathways of health behavior differ when predicting physical conditions and subjective well-being; 3 ) to clarify whether the indirect effects of health literacy are moderated by sex. In addition to providing an updated profile of health literacy level in Hong Kong, the current study also aimed to provide valuable insights for tailoring educational programs to promote general public health.

\section{Methods Design}

A cross-sectional study was conducted with structured questionnaires among individuals living in different districts of Hong Kong. For younger and mid-age adults, the questionnaires were self-administered. For those aged 65 or above, the questionnaires were administered by a trained research assistant, in case they may have difficulties in understanding the questions due to the relatively low level of education. Written consent was obtained at the beginning of the study.

\section{Sample}

A random sampling method was used to recruit two thousand and two hundred thirty-six adults from universities, community centers, social and health centers for the elderly all over Hong Kong. Participants were invited via invitation letters and emails, and those who met the following inclusion criteria were recruited: 1) aged 18 or above; 2) native Chinese speaker (including both Cantonese and mandarin, with the majority of the participants being Cantonese speakers); 3) no history of cognitive impairment (according to the record provided by the community center which helped with the recruitment). The ethical approval for the study was obtained from the Human Research Ethics Committee of XXX University (blinded for review).

\section{Measures}

Participants' demographic information, including sex ( 0 = male, $1=$ female), age, education ( $0=$ "Primary school or lower", $1=$ "secondary school or above"), marital status $(0=$ single/divorced/widowed; $1=$ married $)$, were collected. Participants also reported their health literacy, health behavior, physical symptoms, and well-being.

Health literacy (HL) was measured by the Chinese version of HLS-EU (HLS-Asian-Q Duong et al. 2018). HLS-Asian-Q includes 47 items assessing the information-processing abilities across three domains of health, i.e., health care, disease prevention, and health promotion. Four types of informationprocessing abilities were evaluated: a) the ability to seek and obtain health information (13 items), b) the ability to understand or comprehend health information (11 items), c) the ability to appraise, interpret, or filter health information (12 items), and d) the ability to communicate or apply the information to maintain and improve health (11 items). Participants were asked to choose from " 1 " (very difficult) to "4" (very easy) when responding to the items, and the response of " 5 " (do not know) was coded as missing. The average score of subscale was generated to indicate the level of HL in different dimensions (van der Heide et al. 2013). For general HL, the mean score of all the items was transformed into an $\mathrm{HL}$ index ranging from 0 to 50 according to the formula suggested by the European Health Literacy Project [I = (Mean -1) * 50/3] (Berens et al. 2016), with an higher score indicating greater HL. Participant were grouped into four levels based on the cutoff value suggested by prior study (Hls-Eu Consortium, 2012): an HL index of 0 to 25 indicates the health literacy is "inadequate", and 26 to 33 indicates "problematic"; an index from 34 to 42 indicates "sufficient", and above 42 indicates "excellent" HL. HLS-Asian-Q has been validated in 6 Asian countries/regions including Taiwan, Indonesia, Kazakhstan, Malaysia, Myanmar, and Vietnam (Duong et al. 2018). The Cronbach Alpha in our sample was 0.98 , suggesting good internal consistency.

Health behavior was measured by three items asking about individual's smoking, drinking, and physical exercise. Since different Likert scale was used for each behavior, the responses were recoded into dichotomous variables indicating whether the participant is a current user of tobacco $(0=$ "non-smoker", $1=$ "smoker"), alcohol ( 0 = "non-user of alcohol", 1 = "alcohol user"), or a frequent exerciser ( 0 = "frequent exerciser, i.e., doing exercise for 30 minutes over 2 times per week"; 1 = "infrequent exerciser"). Three items were combined to generate a total score of health behavior, with higher score indicating more presences of unhealth behaviors, and the total score was used in the pathway analysis.

Individual's well-being was measured by the 5-item World Health Organization well-being index (WHO well-being index, (Heun et al. 2001). It asked participants in the past two weeks, how often they have "felt cheerful and in good spirits", "felt calm and relaxed", "felt active and vigorous", "woken up feeling fresh and rested", and "felt daily life has been filled with interesting things". A 6-point response set was used ("0" = "at no time", and " 5 " = "all of the time"). Total score was the sum of all five items, and a higher score indicating better well-being. Good reliability was indicated by a Cronbach alpha of 0.89 .

The presences of physical symptoms were used to evaluate participants' self-reported physical health (Wong et al. 2006). The presence of 28 physical symptoms and chronic disease were measured, namely hypertension, high blood cholesterol, high blood lipid, diabetes, cardiovascular disease, heart failure, respiratory disease, asthma, thyroid disease, liver disease, rheumatism, arthritis, osteoporosis, other musculoskeletal disease, cancer, depression, anxiety disorder, mood disorder, other mental health problem, eating disorder, alcoholism, drug abuse, reproductive disease, hearing impairment, visual impairment, limb loss, and other. The total number of "yes" to the health symptoms was obtained to indicate health condition.

Page $3 / 13$ 


\section{Statistical analyses}

The Lavaan package in $\mathrm{R}$ was used to conduct the structural equation modeling (SEM, Rosseel 2012), to test the hypotheses regarding how different dimensions of health literacy influence physical health and subjective well-being, as well as whether health behavior would mediate the associations if there is any. The reason why we choose to use SEM is because that two outcomes, physical symptoms, and subjective well-being were positively correlated with each other, and when including them in the pathway model, the correlation should be considered. Age, marital status, and education level were controlled as covariates. Pair-wise deletion was used to deal with the missing values in the dataset, which only omits specific variables with missing data on an analysis by analysis basis, to maximize the available data. The scores of four $\mathrm{HL}$ capacities were entered as predictors, with the presence of physical symptoms and subjective well-being index as outcome variables. Sex was also included in the pathway between health literacy capacities to health behavior, to test this moderated mediation effect. The hypothesized model was displayed in Figure 1.

\section{Results}

Two thousand and two hundred thirty-six adults (aged from 18 to 93 , mean $=45.07 \pm 19.05$ ) participated in the survey. Approximately half of the participants were female (53.8\%) and married (55.1\%), with the majority having secondary education or above (79.7\%, for details, see Table 1$)$. The average number of reported physical symptoms was $1.20(S D=1.56)$, and the total score of well-being was $14.84(S D=4.85)$. The average number of unhealthy behaviors are $0.91(S D=0.90)$. By adopting the formula to transform the $\mathrm{HL}$ rating, the average $\mathrm{HL}$ score was $31.24(\mathrm{SD}=8.61)$. Based on this score, participants were categorized into four groups of $\mathrm{HL}, 20.7 \%$ were in the inadequate $\mathrm{HL}$ group, $35.2 \%$ in problematic $\mathrm{HL}$ group, $34.5 \%$ in sufficient $\mathrm{HL}$ group, and $9.6 \%$ reported an excellent level of HL (see Table 1). Since gender was proposed as a moderator, we have tested the gender difference in having limited $\mathrm{HL}$ (inadequate and problematic) and adequate $\mathrm{HL}$ (sufficient and excellent), and the results showed that there were more women being in the limited $\mathrm{HL}$ group compared with men $\left(\chi^{2}=4.23, p=0.04\right)$. Across four domains of $\mathrm{HL}$, evaluating information was perceived as the most difficult (mean per item was $\left.2.76, \mathrm{SD}=0.59\right)$, while applying information was perceived as the easiest (mean per item was $2.95, \mathrm{SD}=0.52$ ).

Table 1

The descriptive results of demographic information, health literacy, health behaviors, and health

\begin{tabular}{|c|c|c|c|}
\hline & Mean (SD) & Range & No. of Missing \\
\hline Age & $46.10(19.5)$ & [18-93] & 0 \\
\hline Gender (\% of women) & $53.8 \%$ & & 1 \\
\hline $\begin{array}{l}\text { Education } \\
\text { (\% of having secondary education or above) }\end{array}$ & $79.7 \%$ & & 0 \\
\hline Marital status (\% of married) & $55.1 \%$ & & 0 \\
\hline Total number of unhealthy behaviors & $0.91(0.9)$ & & \\
\hline$\%$ of smokers & $23.0 \%$ & & 5 \\
\hline$\%$ of alcohol users & $33.7 \%$ & & 21 \\
\hline$\%$ of non-frequent exercisers & $48.3 \%$ & & 7 \\
\hline No. of health conditions & $1.13(1.56)$ & {$[0-15]$} & 2 \\
\hline Well-being & $14.84(4.85)$ & {$[0-25]$} & 3 \\
\hline Health literacy & $31.25(8.61)$ & {$[0-50]$} & 7 \\
\hline Insufficient HL (\%) & $20.7 \%$ & & \\
\hline Problematic HL (\%) & $35.2 \%$ & & \\
\hline Sufficient HL (\%) & $34.5 \%$ & & \\
\hline \multirow[t]{2}{*}{ Excellent HL (\%) } & $9.6 \%$ & & \\
\hline & Mean per item (SD) & & \\
\hline Finding information & $2.88(0.57)$ & & 11 \\
\hline Understanding information & $2.92(0.55)$ & & 8 \\
\hline Evaluating information & $2.76(0.59)$ & & 14 \\
\hline Applying information & $2.95(0.52)$ & & 8 \\
\hline
\end{tabular}

Note: $\mathrm{SD}=$ standardized deviation; $\mathrm{HL}=$ health literacy 
The correlations among predictors, mediator, moderator, and health outcomes were displayed in Table 2. Path analysis using structural equation modeling approach was performed to examine the goodness-of-fit of the hypothesized path model predicting individual's health outcomes, including total number of physical symptoms and sum score of well-being. The two outcome variables were regressed on individual's age, education, marital status, gender, and $\mathrm{HL}$ capacities (i.e., finding information, understanding information, evaluating information, and applying information). By using the Lavaan package in $\mathrm{R}$, the goodness-of-fit of the hypothesized model was considered good (chi-square value $\chi^{2}=28.163$, degree-of-freedom df $=9, p=0.001, R M S E A=0.03, S R M R=$ $0.005, \mathrm{CFI}=0.988, \mathrm{NNFI}=0.952$ ). The structural relationships with standardized path coefficients among the variables are presented in Fig. 2 (only significant paths were included).

Table 2

The correlation matrix among Health literacy, health behavior, and health outcomes

\begin{tabular}{|c|c|c|c|c|c|c|c|c|c|c|c|}
\hline & Education & $\begin{array}{l}\text { Marital } \\
\text { status }\end{array}$ & Gender & $\begin{array}{l}\mathrm{HL} \\
\text { total }\end{array}$ & $\begin{array}{l}\text { HL_ } \\
\text { finding } \\
\text { info }\end{array}$ & $\begin{array}{l}\mathrm{HL} \\
\text { understanding } \\
\text { Info }\end{array}$ & $\begin{array}{l}\text { HL } \\
\text { evaluating } \\
\text { info }\end{array}$ & $\begin{array}{l}\mathrm{HL}_{-} \\
\text {applying } \\
\text { info }\end{array}$ & $\begin{array}{l}\text { Health } \\
\text { behavior }\end{array}$ & $\begin{array}{l}\text { Physical } \\
\text { symptom }\end{array}$ & $\begin{array}{l}\text { Well- } \\
\text { being }\end{array}$ \\
\hline Age & $-0.614^{* \star}$ & $0.390^{\star *}$ & $0.049^{*}$ & $-0.304^{\star \star}$ & $-0.348^{* \star}$ & $-0.315^{\star *}$ & $-0.248^{\star \star}$ & $-0.213^{\star *}$ & $-0.146^{* \star}$ & $0.553^{* *}$ & $0.080^{\star \star}$ \\
\hline Education & 1 & & & & & & & & & & \\
\hline Marital Status & $-0.111^{\star *}$ & 1 & & & & & & & & & \\
\hline Gender & $-0.106^{\star \star}$ & $-0.047^{*}$ & 1 & & & & & & & & \\
\hline $\mathrm{HL}$ total & $0.366^{* *}$ & $-0.045^{*}$ & -0.023 & 1 & & & & & & & \\
\hline HL_finding info & $0.384^{\star *}$ & $-0.052^{\star}$ & -0.020 & $0.936^{\star *}$ & 1 & & & & & & \\
\hline $\begin{array}{l}\text { HL_understanding } \\
\text { Info }\end{array}$ & $0.364^{\star *}$ & $-0.066^{*}$ & -0.020 & $0.947^{\star *}$ & $0.859^{\star \star}$ & 1 & & & & & \\
\hline $\begin{array}{l}\text { HL_evaluating } \\
\text { Info }\end{array}$ & $0.343^{\star *}$ & 0.016 & -0.040 & $0.927^{\star *}$ & $0.823^{\star *}$ & $0.837^{\star \star}$ & 1 & & & & \\
\hline HL_applying info & $0.269^{\star *}$ & $-0.076^{\star *}$ & -0.002 & $0.909^{\star *}$ & $0.790^{\star *}$ & $0.836^{\star *}$ & $0.782^{\star \star}$ & 1 & & & \\
\hline Health behavior & $0.128^{\star \star}$ & -0.009 & $-0.235^{\star \star}$ & $-0.142^{\star \star}$ & $-0.120^{\star \star}$ & $-0.135^{\star \star}$ & $-0.098^{\star \star}$ & $-0.188^{\star \star}$ & 1 & & \\
\hline $\begin{array}{l}\text { Physical } \\
\text { symptoms }\end{array}$ & $-0.456^{\star *}$ & $0.148^{* *}$ & 0.143 & $-0.370^{\star *}$ & $-0.406^{* *}$ & $-0.374^{\star *}$ & $-0.313^{\star *}$ & $-0.281^{\star *}$ & 0.028 & 1 & \\
\hline Well-being & -0.023 & $0.076^{* *}$ & $0.059^{\star *}$ & $0.253^{\star *}$ & $0.218^{\star *}$ & $0.218^{* *}$ & $0.225^{\star *}$ & $0.285^{\star *}$ & $-0.203^{\star *}$ & $-0.057^{\star *}$ & \\
\hline
\end{tabular}

Note: Edu $=$ education $(0=$ "Primary school or lower", $1=$ "secondary school or above"), marital status $(0=$ single/divorced $/$ widowed; $1=\mathrm{married})$, gender $(0=$ male, $1=$ female), $\mathrm{HL}=$ health literacy, $\mathrm{HL}$ _finding info = Health literacy in finding information, $\mathrm{HL}$ _understanding info = health literacy in understanding information, $\mathrm{HL}$ _evaluating info = health literacy in evaluating information, $\mathrm{HL}$ _applying info = health literacy in applying information; higher score of "health behaviors" indicated more presence of unhealthy behaviors.

“*”: $p<0.05 ; “ \star \star ": p<0.01 ; “ \star \star \star ”: ~ p<0.001$ 
The moderated mediation model of health literacy in predicting physical symptoms

\begin{tabular}{|c|c|c|}
\hline & $\mathrm{B}(95 \% \mathrm{Cl})$ & p-value \\
\hline $\begin{array}{l}\text { Association between HL dimensions and } \\
\text { (DV: Physical symptoms \#; IV: HL dimens }\end{array}$ & & \\
\hline HL_finding information & $-0.569^{\star \star \star}$ & $<0.001$ \\
\hline HL_understanding information & $-0.385^{* *}$ & 0.001 \\
\hline HL_evaluating information & 0.113 & 0.220 \\
\hline HL_applying information & 0.196 & 0.193 \\
\hline \multicolumn{3}{|c|}{$\begin{array}{l}\text { Association between } \mathrm{HL} \text { dimensions and health behaviors } \\
\text { (DV: health behaviors \#; IV: HL dimensions; moderator: gender) }\end{array}$} \\
\hline HL_finding information & -0.180 & 0.070 \\
\hline HL_understanding information & -0.083 & 0.478 \\
\hline HL_evaluating information & 0.144 & 0.125 \\
\hline HL_applying information & $-0.198^{*}$ & 0.047 \\
\hline HL_finding information $\times$ gender & 0.160 & 0.237 \\
\hline HL_understanding information $\times$ gender & -0.003 & 0.984 \\
\hline HL_evaluating information $\times$ gender & 0.059 & 0.631 \\
\hline HL_applying information $\times$ gender & $-0.333^{\star *}$ & 0.010 \\
\hline $\begin{array}{l}\text { Association between health behaviors an } \\
\text { (DV: physical symptoms }{ }^{\#} \text {; IV: health beh }\end{array}$ & & \\
\hline Health behavior & $0.118^{\star \star \star}$ & $<0.001$ \\
\hline \multicolumn{2}{|c|}{ (DV: physical symptoms ${ }^{\# ;}$ mediator: health behaviors) } & \\
\hline Men: HL_finding information & -0.0210 .108 & \\
\hline HL_understanding information & -0.010 & 0.485 \\
\hline $\mathrm{HL}$ evaluating information & 0.017 & 0.155 \\
\hline HL_applying information & -0.023 & 0.088 \\
\hline Total effect & $-0.583^{\star \star \star}$ & $<0.001$ \\
\hline
\end{tabular}

\# Physical symptom was adjusted for age, education, and marital status.

DV: dependent variable of the underlying regression model

IV: independent variable of the underlying regression model

B: regression coefficient

Cl: confidence interval

$\mathrm{HL}$ : Health literacy 


\begin{tabular}{|lcc|}
\hline & B (95\% Cl) & p-value \\
\hline Women: HL_finding information & -0.002 & 0.829 \\
\hline HL_understanding information & -0.010 & 0.415 \\
\hline HL_evaluating information & $0.024^{\star}$ & 0.035 \\
\hline HL_applying information & $-0.063^{* \star}$ & 0.001 \\
\hline Total effect & $-0.597^{\star \star *}$ & $<0.001$ \\
\hline \# Physical symptom was adjusted for age, education, and marital status. & & \\
\hline DV: dependent variable of the underlying regression model & & \\
\hline IV: independent variable of the underlying regression model & & \\
\hline B: regression coefficient & & \\
\hline Cl: confidence interval & & \\
\hline HL: Health literacy & & \\
\hline
\end{tabular}




\begin{tabular}{|c|c|c|}
\hline & $\mathrm{B}(95 \% \mathrm{Cl})$ & p-value \\
\hline $\begin{array}{l}\text { Association between HL dimensions and } \\
\text { (DV: well-being \#; IV: HL dimensions) }\end{array}$ & & \\
\hline HL_finding information & 0.399 & 0.280 \\
\hline HL_understanding information & -0.632 & 0.133 \\
\hline HL_evaluating information & 0.283 & 0.395 \\
\hline HL_applying information & $2.564^{\star \star \star}$ & $<0.001$ \\
\hline \multicolumn{2}{|c|}{ (DV: health behaviors\#; IV: HL dimensions; moderator: gender) } & \\
\hline HL_finding information & -0.180 & 0.070 \\
\hline HL_understanding information & -0.083 & 0.478 \\
\hline HL_evaluating information & 0.144 & 0.125 \\
\hline HL_applying information & $-0.198^{*}$ & 0.047 \\
\hline HL_finding information $\times$ gender & 0.160 & 0.237 \\
\hline HL_understanding information $\times$ gender & -0.003 & 0.984 \\
\hline HL_evaluating information $\times$ gender & 0.059 & 0.631 \\
\hline $\mathrm{HL} \_$applying information $\times$gender & $-0.333^{\star *}$ & 0.010 \\
\hline $\begin{array}{l}\text { Association between health behaviors an } \\
\text { (DV: well-being \#; IV: health behaviors) }\end{array}$ & (DV: well-being ${ }^{\# ;}$ IV: health behaviors) & \\
\hline Health behavior & $-0.729^{\star \star \star}$ & $<0.001$ \\
\hline \multicolumn{3}{|l|}{ (DV: well-being \#; mediator: health behaviors) } \\
\hline Men: HL_finding information & \multicolumn{2}{|l|}{0.1310 .088} \\
\hline HL_understanding information & 0.061 & 0.480 \\
\hline HL_evaluating information & -0.105 & 0.136 \\
\hline HL_applying information & 0.144 & 0.067 \\
\hline Total effect & $2.845^{\star \star \star}$ & $<0.001$ \\
\hline Women: HL_finding information & 0.014 & 0.828 \\
\hline HL_understanding information & 0.063 & 0.408 \\
\hline HL_evaluating information & $-0.149^{*}$ & 0.018 \\
\hline HL_applying information & $0.387^{\star \star *}$ & $<0.001$ \\
\hline Total effect & $2.931^{\star \star \star}$ & $<0.001$ \\
\hline \multicolumn{3}{|c|}{ \# Well-being was adjusted for age, education, and marital status. } \\
\hline \multicolumn{3}{|c|}{ DV: dependent variable of the underlying regression model } \\
\hline \multicolumn{3}{|c|}{ IV: independent variable of the underlying regression model } \\
\hline \multicolumn{3}{|l|}{ B: regression coefficient } \\
\hline \multicolumn{3}{|l|}{$\mathrm{Cl}$ : confidence interval } \\
\hline HL: Health literacy & & \\
\hline
\end{tabular}

In consistent with previous literature, health literacy capacities have direct effects on health outcomes. After adjusting for education, age, and marital status, higher level of $\mathrm{HL}$ in finding and understanding information was associated with fewer physical symptoms (HL_finding information: $\beta=-0.569, p<.001$; HL_understanding information: $\beta=-0.385, p=.001$ ); and higher level of health literacy in applying information was associated with greater subjective well- 
being $(\beta=2.564, p<.001)$. In addition, health behaviors (i.e., the number of unhealthy lifestyle behaviors) was also found to mediate the effect of health literacy on physical health and wellbeing. In particular, the capacities of finding and applying health-related information was associated with fewer unhealthy behaviors (HL_finding information: $\beta=-0.180, p=0.07$; HL_applying information: $\beta=-0.198, p=0.05$ ), with the effect of HL_applying information being moderated by gender $(\beta=-0.333, p=0.01)$. Meanwhile, more unhealthy behaviors were related with more physical symptoms $(\beta=0.118, p<.001)$ and lower subjective well-being $(\beta=-0.729, p<.001)$.

To probe the moderating effect of gender, the indirect effects of health literacy in applying information were compared among men and women. The results showed that among men, the capacity of applying information has marginally significant indirect effects on physical health and well-being (physical health: $\beta=-0.023, p=0.088$; well-being: $\beta=0.144, p=0.067$ ). However, among women, the indirect effects on physical symptoms and well-being are both significant, such that via reducing unhealth behaviors, the capacity of applying information was associated with fewer physical symptoms $(\beta=-0.063, p=0.001)$ and greater well-being $(\beta=0.387, p<0.001)$.

\section{Discussion}

Health literacy was found to be a key contributor to individual's health. Although the related changes in health behaviors were proposed to be an underlying mechanism of the health literacy, limited evidence has been found regarding the mediating role of health behavior. By conducting a large-scale survey in Hong Kong, the current study has tested a moderated mediation model of health literacy predicting physical health and well-being through influencing health behaviors. Furthermore, we have looked at the effects of specific health literacy skills, i.e., finding, understanding, evaluating, and applying health-related information.

With a sample of 2236 adults, we found the prevalence of limited HL was $55 \%$, which was close to the average levels in Malaysia and Singapore (Rajah et al. 2019). The prevalence of limited HL was higher among women than men, which was inconsistent with the previous findings that men actually have lower level of HL (Kaneko and Motohashi 2007; Sudore et al. 2006). It might be because that men's education level was higher in our sample $\left(\chi^{2}=24.99, p<0.001\right)$, which was associated with better health literacy. In fact, with logistic regression, it showed that although female are 1.19 times more likely to have limited $\mathrm{HL}$ than male $(p=0.04)$, when education entering the model, the gender effect became insignificant, and people with lower education are 2.47 times more likely to report limited $\mathrm{HL}(p<0.001)$. It is also possible that men may use over-reporting style when answering health literacy questions, thus leading to a higher $\mathrm{HL}$ score (Lee et al., 2013b). The structural modeling analysis showed similar patterns of health literacy in predicting physical health and subjective well-being. While different health literacy capacities had different direct and indirect effects on health behaviors and outcomes. The health literacy in finding and understanding information showed a direct effect in predicting fewer physical symptoms, and health literacy in applying information was directly associated with greater well-being. As for the indirect effect via health behavior, only health literacy in applying information showed a significant result, which was moderated by sex. Despite similar patterns were found, the indirect effect of the capacity in applying information on promoting health and well-being was only significant among women.

This is the first study to test how the direct and indirect effects of health literacy vary across the four dimensions. In line with a previous study on college students showing that understanding and applying health information had stronger effect in promoting people's smoking cessation (Panahi et al. 2018), our findings suggested that finding and understanding information were associated with fewer self-report physical symptoms, while applying information had a moderated mediation effect, that only among women the indirect positive effect reached significant level. The results suggested that the capacities of finding and understanding information may influence our health in a more direct and general way, while the applying health information may function via modifying our behavior, particularly for women. It is probably because applying information (e.g., "make decisions to improve your health", "Join a sport club or exercise class") is usually the last step when making health decision, which is more closely related to taking action. Therefore, this is the only domain that had an indirect effect on health via behaviors. Interestingly, the capacity of evaluating information didn't show any effect on physical symptoms or well-being, which is consistent with previous findings that smoking cessation was associated with health literacy capacities except evaluating health information (Panahi et al. 2017). It is possible that evaluating information was usually perceived as the most difficult (e.g., Nutbeam, 2000; Zhang, Or, \& Chung, 2020), thus it is considered as a more advanced capacity, and would not show immediate effects on individual's health behaviors or outcomes.

The mediating role of health behaviors is consistent with the previous pathway model linking health literacy to health outcome in the patient population, which suggested that health literacy functions mainly in three domains, including the access to and use of health care, patient-provider interaction, and patient's selfcare (Osborn et al. 2011; Paasche-Orlow and Wolf 2007). The health behaviors that we have focused on fell into the domain of self-care. In line with previous studies showing that health literacy is associated with being physically active, taking balanced diet, and lower usage of tobacco and alcohol (Geboers et al. 2016; Liu et al. 2018), our study also showed that across different age groups, the health literacy, especially better ability in applying health information, would reduce unhealth behaviors thus benefiting physical health and well-being via promoting self-care agency. Furthermore, this indirect effect of health literacy only reached significance level among women, potentially suggesting that with sufficient health information, women could more readily apply them to modify their behaviors toward a healthier lifestyle. Men, on the other hand, may need to be motivated to transform the health information they have into actions.

Though our findings provided evidence for how health literacy influences health status and well-being through behaviors, there are several limitations to be acknowledged. First, the health status was measured by self-reported physical symptoms, which could be influenced by individual's personal reporting styles, and may not accurately capture the health condition of the individual. Future studies should adopt more objective measures, such as medical record or physiological measures to see if the influences of health literacy will still be found. Second, we have only collected cross-sectional data in the current study, which may not be sufficient to support the causal relationship between health literacy and health outcomes, in other words, there might be a reciprocal association between the two. For example, a previous study has found that people with chronic disease may have higher level of health literacy, which would prevent them from developing more chronic symptoms (Qian et al. 2019). Therefore, longitudinal data is needed to further clarify the interplay between health literacy and health outcomes. 


\section{Conclusion}

To conclude, the current study provided supportive evidence that promotions for health literacy is still urgent for Hong Kong population, such that over $50 \%$ of the lay public showed a limited level of health literacy, and this prevalence is a little higher among women. In addition, by addressing specific direct and indirect effects of four dimensions of health literacy on physical health and well-being, the findings showed that better perceived capacities in finding and understanding health information could lead to better physical health, while better capacity in applying health information is associated with healthier lifestyle. Based on the findings, more educational programs to advocate health knowledge and increase awareness of healthy lifestyle should be developed for the lay public. In particularly, effort should be paid to enhance women's general health literacy, and to motivate men to apply their health knowledge in behavior modifications.

\section{Abbreviations}

Agency for Healthcare Research and Quality (AHRQ)

Health literacy $(\mathrm{HL})$

HL_finding info: Health literacy in finding information

HL_understanding info= health literacy in understanding information

HL_evaluating info= health literacy in evaluating information

HL_applying info= health literacy in applying information

\section{Declarations}

\section{Ethics approval and consent to participate}

The ethical approval for the study was obtained from the Human Research Ethics Committee of The Education University of Hong Kong, and participants have provided written consent before taking part in the study.

\section{Consent to publish}

Not applicable

\section{Availability of data and materials}

Derived data supporting the findings of the study is available from the corresponding author on request.

\section{Competing interests}

There was no competing interest that need to be declared.

\section{Funding}

This study was funded through Dean's Strategies Research Area (grant number 04187). The funder has no role in study design, data collection and analysis, or preparation of manuscript

\section{Authors' Contributions}

ZF has analyzed and interpreted the data, as well as written and edited the manuscript. PO was a major contributor in generating the research idea, collecting data, and editing the manuscript. JC was also a contributor in generating the research idea and data collection.

All authors have read and approved the final version of the manuscript.

\section{Acknowledgements}

The author would like to thank Prof. Cheng Sheung-Tak for his constructive suggestions and help for the manuscript.

\section{References}

Angner, E., Miller, M. J., Ray, M. N., Saag, K. G. \& Allison, J. J. (2010). Health literacy and happiness: A community-based study. Social Indicators Research, 95, 325

Baker, D. W., Wolf, M. S., Feinglass, J., Thompson, J. A., Gazmararian, J. A. \& Huang, J. (2007). Health literacy and mortality among elderly persons. Archives of Internal Medicine, 167, 1503-1509 
Baker, D. W., Gazmararian, J. A., Williams, M. V., Scott, T., Parker, R. M., Green, D., Ren, J. \& Peel, J. (2002). Functional health literacy and the risk of hospital admission among Medicare managed care enrollees. American Journal of Public Health, 92, 1278-1283

Berens, E., Vogt, D., Messer, M., Hurrelmann, K. \& Schaeffer, D. (2016). Health literacy among different age groups in Germany: results of a cross-sectional survey. BMC public health, 16, 1151

Berkman, N. D., Sheridan, S. L., Donahue, K. E., Halpern, D. J. \& Crotty, K. (2011). Low health literacy and health outcomes: an updated systematic review. Annals of Internal Medicine, 155, 97-107

Bird, C. E. \& Rieker, P. P. (1999). Gender matters: an integrated model for understanding men's and women's health. Social science \& medicine, $48,745-755$

Blow, F. C., Walton, M. A., Barry, K. L., Coyne, J. C., Mudd, S. A. \& Copeland, L. A. (2000). The relationship between alcohol problems and health functioning of older adults in primary care settings. Journal of the American Geriatrics Society, 48, 769-774

Coffman, M. J. \& Norton, C. K. (2010). Demands of immigration, health literacy, and depression in recent Latino immigrants. Home Health Care Management \& Practice, 22, 116-122

Davey, J., Holden, C. A. \& Smith, B. J. (2015). The correlates of chronic disease-related health literacy and its components among men: a systematic review. BMC Public Health, 15, 589

Duong, T., Sørensen, K., Pelikan, J. M., Van den Broucke, S., Lin, I., Lin, Y., Huang, H. \& Chang, P. W. (2018). Health-related behaviors moderate the association between age and self-reported health literacy among Taiwanese women. Women \& health, 58, 632-646

Gao, C., Qiao, X., Li, G., Zhai, J., Si, J., Zhao, C. \& Huang, S. (2018). The prevalence of health literacy of Chinese residents for infectious disease between 2011 and 2015: findings from a meta-analysis. TMR Integrative Nursing, 2, 76-86

Geboers, B., Reijneveld, S. A., Jansen, C. J. \& de Winter, A. F. (2016). Health literacy is associated with health behaviors and social factors among older adults: Results from the LifeLines Cohort Study. Journal of health communication, 21, 45-53

Geboers, B., de Winter, A. F., Luten, K. A., Jansen, C. J. \& Reijneveld, S. A. (2014). The association of health literacy with physical activity and nutritional behavior in older adults, and its social cognitive mediators. Journal of health communication, 19, 61-76

Heun, R., Bonsignore, M., Barkow, K. \& Jessen, F. (2001). Validity of the five-item WHO Well-Being Index (WHO-5) in an elderly population. European archives of psychiatry and clinical neuroscience, 251, 27-31

HIs-Eu Consortium (2012). Comparative report of health literacy in eight EU member states. The European health literacy survey HLS-EU

Kaneko, Y. \& Motohashi, Y. (2007). Male gender and low education with poor mental health literacy: a population-based study. Journal of epidemiology, 17, $114-119$

Koenig, V. E. (2019). Health Literacy: A Universal Call to Action. Journal of Psychology and Mental Health Care, 10. DOI 10.31579/2637-8892.19/011.

Lee, S. D., Tsai, T. \& Tsai, Y. (2013a). Accuracy in self-reported health literacy screening: a difference between men and women in Taiwan. BMJ open, 3 , e002928

Lee, S. D., Tsai, T. \& Tsai, Y. (2013b). Accuracy in self-reported health literacy screening: a difference between men and women in Taiwan. BMJ open, 3 , e002928

Lincoln, A., Paasche-Orlow, M. K., Cheng, D. M., Lloyd-Travaglini, C., Caruso, C., Saitz, R. \& Samet, J. H. (2006). Impact of health literacy on depressive symptoms and mental health-related: quality of life among adults with addiction. Journal of general internal medicine, 21, 818-822

Liu, Y., Xue, L., Xue, H. \& Hou, P. (2018). Health literacy, self-care agency, health status and social support among elderly Chinese nursing home residents. Health education journal, 77, 303-311

Mokdad, A. H., Marks, J. S., Stroup, D. F. \& Gerberding, J. L. (2004). Actual causes of death in the United States, 2000. Jama, 291, 1238-1245

Murray, M. D., Wu, J., Tu, W., Clark, D. O., Weiner, M., Morrow, D. G. \& Brater, D. C. (2004). Health literacy predicts medication adherence. Clinical Pharmacology \& Therapeutics, 75, P76

Osborn, C. Y., Paasche-Orlow, M. K., Bailey, S. C. \& Wolf, M. S. (2011). The mechanisms linking health literacy to behavior and health status. American Journal of Health Behavior, 35, 118-128

Paasche-Orlow, M. K. \& Wolf, M. S. (2007). The causal pathways linking health literacy to health outcomes. American Journal of Health Behavior, 31, S19-S26

Panahi, R., Ramezankhani, A., Tavousi, M., Osmani, F. \& Niknami, S. (2017). Which Health Literacy Skills Are Associated with Smoking?. Zahedan Journal of Research in Medical Sciences, 19 
Panahi, R., Ramezankhani, A., Haerimehrizi, A., Tavousi, M., Darestani, M. K. \& Niknami, S. (2018). Which dimensions of Health Literacy predict the adoption of smoking preventive Behaviors?. Journal of Health in the Field, 5

Qian, X., Chen, Z. \& He, T. (2019). Health literacy and its effect on chronic disease prevention: evidences from China's national health literacy surveillance data Rajah, R., Hassali, M. \& Murugiah, M. K. (2019). A systematic review of the prevalence of limited health literacy in Southeast Asian countries. Public health, $167,8-15$

Reisi, M., Javadzade, S. H., Heydarabadi, A. B., Mostafavi, F., Tavassoli, E. \& Sharifirad, G. (2014). The relationship between functional health literacy and health promoting behaviors among older adults. Journal of education and health promotion, 3

Rosseel, Y. (2012). Lavaan: An R package for structural equation modeling and more. Version 0.5-12 (BETA). Journal of statistical software, 48, 1-36

Schillinger, D., Grumbach, K., Piette, J., Wang, F., Osmond, D., Daher, C., Palacios, J., Sullivan, G. D. \& Bindman, A. B. (2002). Association of health literacy with diabetes outcomes. Jama, 288, 475-482

Schünemann, J., Strulik, H. \& Trimborn, T. (2017). The gender gap in mortality: How much is explained by behavior?. Journal of health economics, 54, 79-90

Södergren, M., McNaughton, S. A., Salmon, J., Ball, K. \& Crawford, D. A. (2012). Associations between fruit and vegetable intake, leisure-time physical activity, sitting time and self-rated health among older adults: cross-sectional data from the WELL study. BMC Public Health, 12, 551

Sørensen, K., Van den Broucke, S., Fullam, J., Doyle, G., Pelikan, J., Slonska, Z. \& Brand, H. (2012). Health literacy and public health: A systematic review and integration of definitions and models. BMC public health, 12, 80

Sudore, R. L., Mehta, K. M., Simonsick, E. M., Harris, T. B., Newman, A. B., Satterfield, S., Rosano, C., Rooks, R. N., Rubin, S. M. \& Ayonayon, H. N. (2006). Limited literacy in older people and disparities in health and healthcare access. Journal of the American Geriatrics Society, 54, 770-776

Suka, M., Odajima, T., Okamoto, M., Sumitani, M., Igarashi, A., Ishikawa, H., Kusama, M., Yamamoto, M., Nakayama, T. \& Sugimori, H. (2015). Relationship between health literacy, health information access, health behavior, and health status in Japanese people. Patient education and counseling, $98,660-668$

van der Heide, I., Rademakers, J., Schipper, M., Droomers, M., Sørensen, K. \& Uiters, E. (2013). Health literacy of Dutch adults: a cross sectional survey. BMC public health, 13,179

Von Wagner, C., Steptoe, A., Wolf, M. S. \& Wardle, J. (2009). Health literacy and health actions: a review and a framework from health psychology. Health Education \& Behavior, 36, 860-877

Wong, T. K., Chung, J. W., Fan, K. L., Chow, M. W., Lau, Y. K., Cheung, C. C., Au, A. Y. \& Ma, P. M. (2006). Experience sharing on the implementation of telehealth system in Hong Kong. Studies in health technology and informatics, 122, 654-656

Zhang, F., Or, P. P. \& Chung, J. W. (2020). The effects of health literacy in influenza vaccination competencies among community-dwelling older adults in Hong Kong. BMC geriatrics, 20, 1-7

\section{Figures}

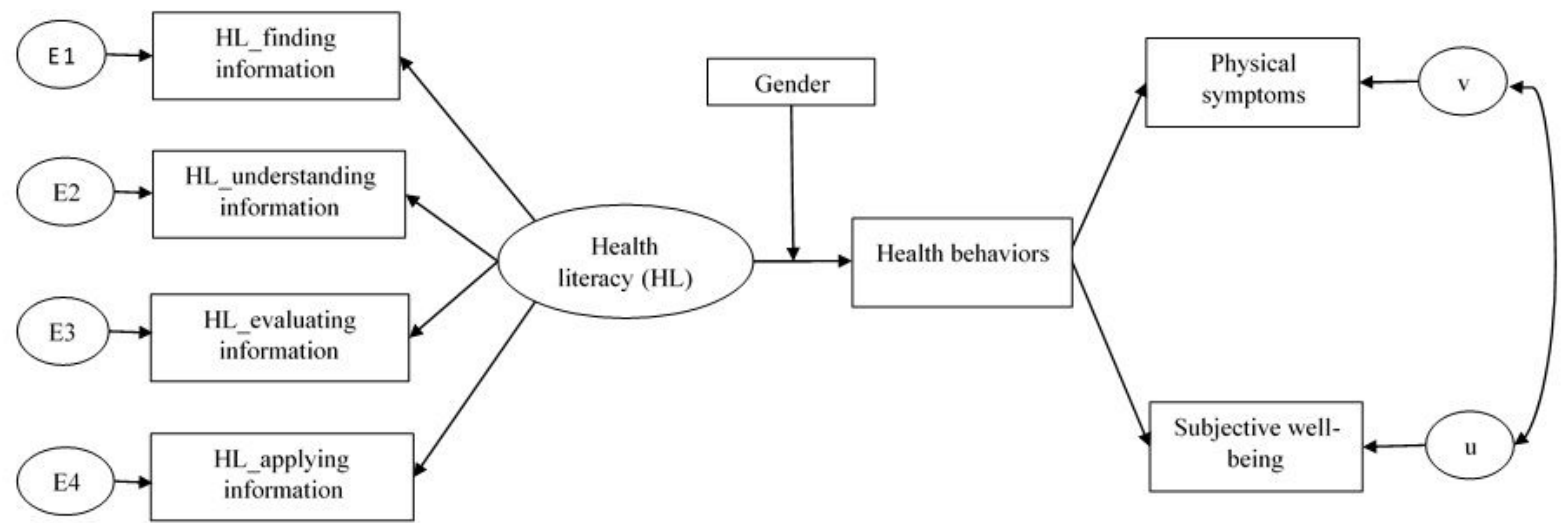

Figure 1

Page $12 / 13$ 


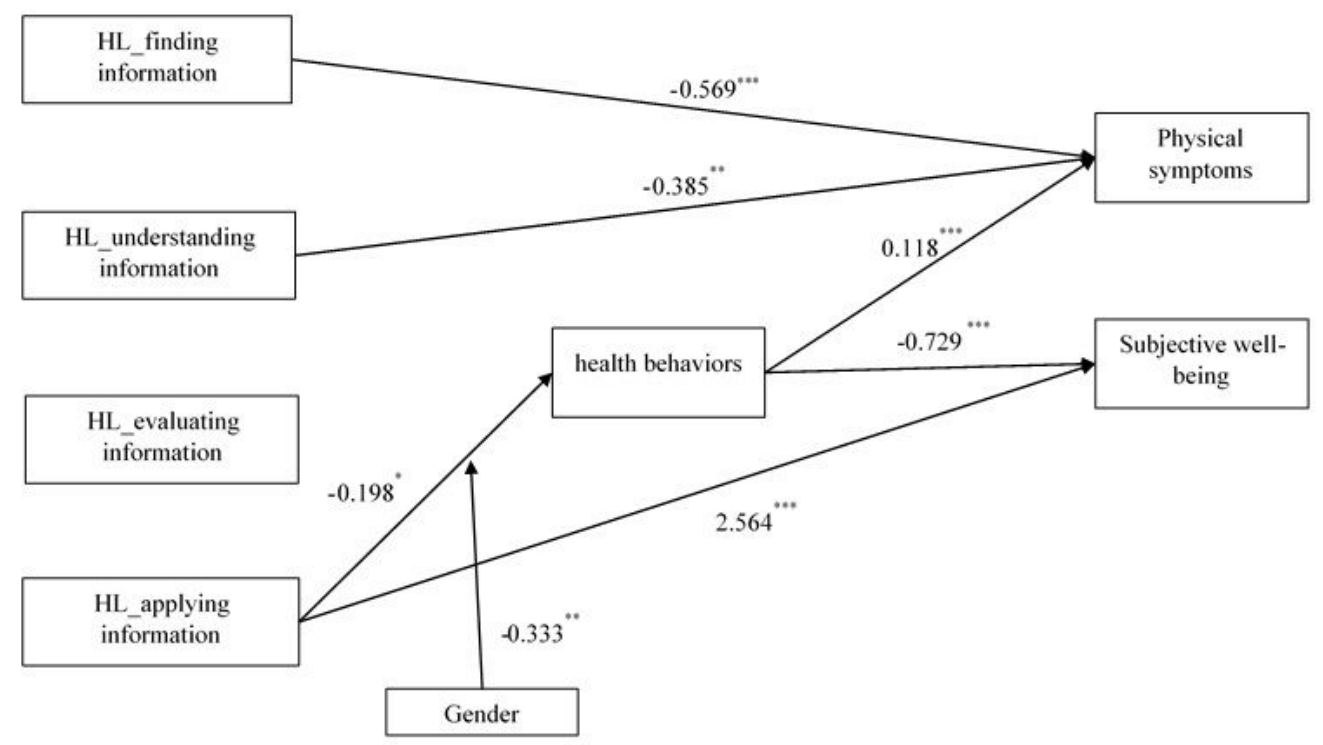

Figure 2

The structural model of health literacy predicting physical health and well-being (Note: health behavior, physical symptom and well-being were adjusted for individual's age, educational level, and marital status)

\section{Supplementary Files}

This is a list of supplementary files associated with this preprint. Click to download.

- STROBEchecklistcrosssectional.doc 